\title{
LA PROBABILIDAD COMO GRADO DE POSIBILIDAD
}

\author{
José LUIS ROLLERI \\ Facultad de Filosofía \\ Universidad Autónoma de Querétaro \\ jlrolleri@hotmail.com
}

En este siglo, [...] una nueva modalidad — una posibilidad con grados - ha tomado un lugar central en el escenario de la ciencia física: la probabilidad.

Van Fraassen 1980

RESUMEN: En desacuerdo crítico con la interpretación propensiva popperiana, sostengo la tesis de que el concepto matemático de probabilidad puede ser interpretado como el grado de posibilidad física.

Sea el suceso singular $e$ el estado final de la evolución de un sistema físico $S$, dado un estado inicial específico de $S$, entonces $e$ es físicamente posible, relativamente a una teoría $\mathbf{T}$, si el estado inicial de $S$ es descriptible por $\mathbf{T}$ y la descripción del suceso $e$ es prescriptible por las leyes de $\mathbf{T}$.

En la teoría cuántica, el carácter objetivo de esta propuesta radica en que las descripciones probabilistas cuánticas son irreductibles a descripciones clásicas con variables ocultas locales. Sustento esta interpretación mostrando que hay una representación implícita de enunciados probabilistas cuánticos referentes a los resultados posibles de procesos experimentales de observables con un espectro discreto de valores propios.

PALABRAS CLAVE: interpretación, propensión, representación

SUMMARY: In critical disagreement with Popper's propensity interpretation, I hold the thesis that the mathematical concept of probability can be interpreted as the degree of physical possibility.

Let a single event $e$ be the final state of the evolution of a physical system $S$, given a specific initial state of $S$, then $e$ is physically possible, relative to a theory $\mathbf{T}$, if the initial state of $S$ is describable by $\mathbf{T}$ and the description of the event $e$ is prescriptible by the laws of $\mathbf{T}$.

In quantum theory, the objective character of this proposal lies in the fact that quantum probabilistic descriptions cannot be reduced to classical descriptions with local hidden variables. In support of this interpretation, I show that there is an implicit representation of quantum-mechanics probabilistic statements, that refer to the possible outcomes in experimental process of observables with a discrete spectrum of eigenvalues.

KEY WORDS: interpretation, propensity, representation 


\section{Introducción}

Una interpretación de la probabilidad pretende dar respuesta a la pregunta ¿qué es la probabilidad? en el marco de la teoría de las probabilidades. Más específicamente, el problema que se intenta solucionar consiste en interpretar (especificar el significado de) enunciados probabilistas de manera acorde con las propiedades formales que los axiomas de la teoría matemática les atribuyen, y considerando esta última como un cálculo abstracto. Así, lo que se requiere es, principalmente, especificar el significado de la función de probabilidad que ocurre en los enunciados probabilistas.

La interpretación que predomina actualmente en el campo de la filosofía de la ciencia, desde que Popper la propuso como una alternativa a la frecuencial, ${ }^{1}$ es la interpretación propensista. Esta interpretación intenta elucidar el concepto de probabilidad con base en una noción de propensión física; esto es, pretende especificar el significado de las probabilidades atribuibles a sistemas físicos en términos de propensiones.

En este artículo me ocupo de este problema, y propongo una interpretación, alternativa a la propensista, de la teoría de las probabilidades. A grandes rasgos, la propuesta que presento consiste en (1) caracterizar una noción de "físicamente posible", aplicable a sucesos físicos singulares, relativamente a una teoría física, y (2) interpretar los enunciados de probabilidad como expresiones del grado de posibilidad física de esos sucesos.

La idea básica que adopto para caracterizar esa noción de posibilidad física es que cada teoría física particular especifica los sucesos que son posibles al describir los estados de los sistemas que estudia, y los cambios de estado o evoluciones de esos sistemas, prescritos por las leyes de la teoría. Para ello, ofrezco una noción general de suceso físicamente posible, relativamente a $\mathbf{T}$, donde $\mathbf{T}$ es una variable para teorías físicas, particularmente, para teorías con estructura probabilista.

Con esto se consigue interpretar los enunciados de probabilidad que una teoría física asevera si se concibe, como propongo,

${ }^{1}$ Véanse Popper 1957 y 1959. 
que estos enunciados son las expresiones cuantitativas de las posibilidades físicas de los sucesos que establece esa teoría. Es decir, el significado de un enunciado probabilista de una teoría $\mathbf{T}$ referido a un suceso singular $s$, descrito por $\mathbf{T}$, consiste en expresar de manera cuantitativa el grado en que $s$ es físicamente posible según las leyes de $\mathbf{T}$.

La interpretación que aquí propongo pretende dar cuenta de las probabilidades de una manera no epistémica. Esto puede establecerse en referencia a los enunciados probabilistas de la mecánica cuántica en virtud de los resultados de los experimentos tipo Einstein, Podolsky y Rosen que, en 1982, llevaron a cabo Alain Aspect y colaboradores, y en los cuales se constata la irreductibilidad de las probabilidades cuánticas. Lo que nos permite atribuir un carácter objetivo a esas probabilidades y, de ahí, a las posibilidades físicas correspondientes.

Antes de exponer la interpretación de la probabilidad que propongo, discutiré críticamente las interpretaciones propensistas de Karl Popper, Ronald Giere y Patrick Suppes, para mostrar que pretenden elucidar el concepto de propensión recurriendo a una noción no aclarada de posibilidad (no lógica); que la noción que realmente usan en sus respectivas interpretaciones es esta última (su recurso a la noción de propensión es más bien verbal, declarativo); y que, por ello, para el propósito de interpretar las probabilidades, dicho concepto resulta prescindible y eliminable. ${ }^{2}$ Después presentaré la noción de "posibilidad física", aplicable a sucesos singulares, en términos de la cual interpreto los enunciados probabilistas de teorías físicas particulares como la expresión cuantitativa del grado de posibilidad física de esos sucesos. Al final, con el propósito de dar soporte a la interpretación propuesta, muestro que existe una representación implícita de cierto tipo de probabilidades cuánticas en términos de posibilidades físicas.

${ }^{2}$ Para otras dificultades e insuficiencias de esta interpretación, véanse Salmon 1979 y Humphreys 1985. 


\section{La noción de propensión física}

Para los propensistas, al menos para Popper y Giere, las propensiones son propiedades relacionales de los sistemas físicos. En Popper, ciertos dispositivos experimentales (situaciones físicas) ${ }^{3}$ entrañan las condiciones generadoras de las propensiones, y éstas son propiedades tanto de los dispositivos (p. ej., un lanzador de dados), como de algunos objetos (los dados). (Véase 1982a, pp. 398 y 399.)

Por ello, Popper asigna probabilidades a sistemas físicos compuestos por un aparato experimental (p. ej., el lanzador de monedas), y unos objetos físicos (la moneda y la superficie sobre la que cae), y mantiene que estos sistemas, llamados dispositivos experimentales, generan las propensiones.

Además, Popper sostiene que las propensiones son entidades físicas que, aunque inobservables - como las fuerzas newtonianas - , son cognoscibles por ser medibles a través de frecuencias relativas. Así, él afirma que

lo que propongo es una nueva hipótesis física (o quizá una hipótesis metafísica), análoga a la hipótesis de las fuerzas newtonianas. Es la hipótesis según la cual todo dispositivo experimental (y, por tanto, todo estado de un sistema) genera propensiones que a veces pueden ser contrastadas por medio de frecuencias. Esta hipótesis es contrastable y está corroborada por ciertos experimentos cuánticos. (1982a, p. 399)

Asimismo, él pretende interpretar la teoría de la probabilidad objetivamente, porque concibe las propensiones como posibilidades físicas, las cuales son propiedades reales de sistemas físicos:

${ }^{3}$ En respuesta a una crítica señalada por Bunge, Feyerabend y Jammer de que su insistencia en hablar de "dispositivos experimentales" (experimental arrangements) sugiere una posición subjetivista, Popper aclara que su intención fue siempre referirse a situaciones físicas, explicando que: "naturalmente, la situación objetiva será normalmente una que haya surgido en el mundo físico sin interferencia humana, aunque puede ser debida al hombre y quizá incluso a un físico que ha construido un aparato. En este último caso, hablamos de "dispositivo experimental"” (1982c, p. 90). 
Llamando a las medidas de las posibilidades probabilidades objetivas o propensiones, no estoy haciendo nada más que usar otra palabra; pero lo hago para llamar la atención sobre el hecho de que esas "posibilidades" se consideran ahora magnitudes físicas que, como las fuerzas, pueden interactuar y combinarse, y pueden considerarse, a pesar del término "posibilidad", como físicamente reales: no son simplemente posibilidades lógicas, sino que son posibilidades físicas. (Popper 1982b, p. 126)

De tal manera que, para Popper, hay posibilidades inherentes a los dispositivos experimentales a las que corresponden los resultados de los experimentos. Él las llama propensiones concibiéndolas como físicamente reales y considera que las medidas de esas propensiones son probabilidades objetivas ( $c f r$. 1982b, pp. 126 y 127).

Como puede verse en lo anterior, el concepto clave para caracterizar las propensiones es el de posibilidad física. $\mathrm{Y}$ es precisamente este concepto el que permite a Popper decir que hay propensiones inherentes a ciertos sistemas físicos, como los sistemas cuánticos. No obstante, él no intenta dar una explicación ulterior de la noción de posibilidad física sino, más bien, la asume tácitamente como primitiva. ${ }^{4}$

Según Giere, las propensiones son tendencias de algo $(X)$ a producir algo más $(Y)$. La $X$ es un dispositivo azaroso $C S U$ [chance set-up] o experimento aleatorio [random experiment] con un conjunto finito $S$ de resultados posibles. La $Y$ representa un resultado específico $E$ de un ensayo $L$ en un $C S U$. Esto está implícito en el significado físico propuesto por Giere para los enunciados de probabilidad. Si asociamos una distribución de probabilidad al conjunto finito $S$ de resultados posibles de un dispositivo azaroso $C S U$, entonces $\mathrm{P}(E)=r$ significa "La fuerza de la propensión de $C S U$ a producir el resultado $E$ en el ensayo $L$ es $r$ " (Giere 1973, p. 471). Este tipo de enunciados - anota Giere - refiere claramente a ensayos particulares y son

${ }^{4}$ En su última publicación sobre el tema, Un mundo de propensiones, de 1990, Popper reitera su concepción de las propensiones como posibilidades inherentes a situaciones o sistemas físicos e insiste en compararlas con fuerzas newtonianas. 
los enunciados primarios de su interpretación propensiva de casos singulares. Los enunciados $P(E)$ pueden generalizarse, y de ahí se obtiene que: "para todo ensayo de $C S U$, la propensión de $C S U$, a producir el resultado $E$ es $r$ " (Giere 1973, p. 471), lo que expresa que las propensiones son propiedades relacionales del dispositivo azaroso $C S U$ y el resultado posible específico $E$.

Es digno de destacar que los sistemas físicos que Giere llama "dispositivos azarosos" son sistemas indeterministas; esto está implícito en la noción del conjunto finito $S$ de resultados posibles asociado a un CSU. En un artículo posterior (1976), Giere es más explícito en referencia a esta cuestión al afirmar que las propensiones son tendencias causales de sistemas estocásticos. Ahí, Giere caracteriza esos sistemas recurriendo a un concepto, que no aclara ni precisa, de "estado final físicamente posible de un sistema físico", como sigue:

A grandes rasgos, un sistema es determinista si exactamente un estado final está unívocamente determinado por el estado inicial, esto es, dado el estado inicial, un estado final precisamente definido es físicamente posible. Si relativamente a algún estado inicial, más de un estado final es físicamente posible, entonces el sistema es indeterminista. [...] Un sistema indeterminista es probabilista (o estocástico) si cada estado inicial determina una distribución de probabilidad sobre todos los estados finales físicamente posibles. (Giere 1976, p. 327)

Además, él agrega que la idea intuitiva que subyace a su propuesta propensista es que las distribuciones de probabilidad, asociadas a los sistemas indeterministas, son distribuciones de tendencias causales no reductibles a frecuencias relativas.

Para Giere, entonces, el dominio de aplicación de la teoría de la probabilidad está formado por los estados finales físicamente posibles de sistemas físicos indeterministas, estocásticos. Sin embargo, Giere no especifica ni elabora el concepto objetivo de posibilidad física que utiliza, sino que lo presupone. Aunque es tal concepto el que permite a Giere caracterizar algunos sistemas físicos como indeterministas y el que utiliza para afirmar que las propensiones son tendencias causales de sistemas estocásticos 
a adoptar un estado específico dentro de una clase de estados finales físicamente posibles.

Por su parte, Patrick Suppes (1987) intenta subsanar la carencia de teoremas de representación adecuados en la versión popperiana de la interpretación propensista. ${ }^{5}$ En ese trabajo, él ofrece varias definiciones conjuntistas y cuatro teoremas de representación que se refieren a procesos con comportamiento aleatorio - físicos o psicológicos—, que para él son capturables con la noción de propensión o, mejor, que exhiben propensiones. Estos teoremas se aplican, respectivamente, a fenómenos de decaimiento radiactivo, a la fuerza de respuesta en ciertas situaciones psicológicas, a juegos de azar como las ruletas, y a procesos aleatorios que pueden producirse en sistemas deterministas.

Antes que nada, hay que mencionar que Suppes adopta una posición pluralista respecto al problema de la interpretación de las probabilidades, en el sentido de que cree que no hay un único concepto de probabilidad que sea aplicable a cualquier fenómeno aleatorio pertinente, sino que, dependiendo del tipo de fenómeno en cuestión, es apropiado usar o bien un concepto subjetivo de probabilidad o bien uno objetivo; y que, en este último caso, habría fenómenos aleatorios para los cuales es adecuada una interpretación de la probabilidad en términos de propensiones, como el fenómeno de decaimiento radiactivo. En este sentido encuentra Suppes relevante la noción de propensión para capturar formalmente las probabilidades que atribuimos a cierto tipo de fenómenos aleatorios, como el recién señalado.

Sin embargo, por un lado, Suppes no elucida el concepto de propensión que pretende asociar a sus definiciones y teoremas. Por otro, enuncia en su primera definición unos axiomas estructurales generales que definen la noción de "estructura cualitativa de probabilidad" en lugar de un concepto premétrico de propensión; esos axiomas, entonces, no caracterizan formalmente nin-

${ }^{5}$ Un teorema de representación para una interpretación de la teoría de la probabilidad consiste en demostrar que existe una función homomórfica de una estructura cualitativa (en el caso de la interpretación propensista, de una estructura de propensión) a una estructura cuantitativa de probabilidad tal que se cumplan los axiomas de esa teoría matemática. 
guna noción de propensión (véase Suppes 1987, pp. 338 y 339). Respecto a esto, él anota que el lugar propio para caracterizar las propensiones está en los axiomas específicos que expresan las hipótesis físicas acerca de procesos como el decaimiento radiactivo. En este sentido, afirma que el axioma específico que formula para definir la noción de estructura cualitativa de tiempo de espera [waiting-time] con independencia del pasado - que aplica precisamente a procesos de decaimiento radiactivo- es "un axioma especial para ciertos fenómenos físicos. Representa, por lo tanto, una expresión cualitativa de una propensión" (1987, pp. 345 y 346). De esta manera, Suppes no intenta dar explícitamente una noción general de propensión física, dejando el contenido semántico que pudiéramos atribuir a esa noción a los axiomas específicos de sus definiciones. En todo caso, la noción que subyace a su concepto general de "estructura cualitativa de probabilidad" expresamente no es la de propensión sino más bien, como hemos anotado, la de posibilidad o azar [chance] objetivo (cfr. 1987, p. 343). En otra obra, Suppes mismo argumenta a favor de concebir los procesos de decaimiento radioactivo como un caso típico de proceso físico que exhibe una aleatoriedad [randomness] objetiva ( $c f r$. 1984, pp. 22 y 23).

Más específicamente, encuentro que la noción que está implícitamente asociada a los axiomas estructurales generales que definen las estructuras de probabilidad cualitativa de Suppes es la de posibilidad que se atribuye a los sucesos elementos del llamado "espacio de sucesos". Y puesto que Suppes no establece un vínculo entre las pretendidas estructuras de propensión que define y una noción de propensión previamente elucidada, no es claro que las definiciones de esas estructuras capturen de alguna manera un concepto de propensión. ${ }^{6}$

\footnotetext{
${ }^{6}$ Maria Carla Galavotti, al comentar el trabajo de Suppes de 1987, pregunta: "¿el artículo de Suppes se ocupa realmente de las propensiones o es, más bien, acerca de una noción menos específica de 'probabilidad objetiva' o "azar [chance] objetivo'?" (1987, p. 359). Comparto con Galavotti la impresión de que si bien la interpretación propuesta [intended] de Suppes de sus definiciones y teoremas es en términos de propensiones, parece más bien estar representando una noción cualitativa de probabilidad o posibilidad (no lógica).
} 
Hemos mostrado que en esas tres propuestas de interpretación propensista, algún concepto de posibilidad física desempeña un papel importante: en efecto, el papel central de ser el concepto interpretativo del de probabilidad, en lugar de la noción de propensión. ¿Qué podemos concluir de esto? Para el propósito de interpretar el concepto de probabilidad que o bien los propensistas tienen la labor inconclusa de elucidar la noción de propensión, o bien que (al menos en estas tres versiones) esa noción resulta innecesaria y prescindible y que, por el principio de economía ontológica de Ockham, debemos eliminarla.

\section{La probabilidad como posibilidad con grados}

Los conceptos de probabilidad, posibilidad, prospecto, propensión, fortuito, casual, aleatorio, estocástico y azar forman un grupo de nociones de azar [chance] semánticamente ligadas que es parte de nuestro marco conceptual contemporáneo y por medio del cual capturamos intelectualmente, y tratamos de entender, ciertos comportamientos peculiares que exhiben algunos sistemas físicos que estudian teorías científicas, como la mecánica cuántica. ${ }^{7}$ El concepto de probabilidad es un miembro destacado en ese grupo de conceptos de azar porque contamos con una teoría matemática de él, la cual nos permite expresar de manera numérica, dentro del marco de una teoría científica, el comportamiento azaroso de sistemas físicos como los cuánticos.

Como hemos visto, Popper y otros eligen de ese grupo el concepto de propensión para interpretar la probabilidad; sin embargo, en su intento toman tácitamente alguna noción no lógica de posibilidad como básica, sin aclararla ulteriormente. Este modo de proceder es formalmente correcto, aunque presenta la dificultad de que al no salir de ese grupo de conceptos de azar, su interpretación de las probabilidades en términos, en última instancia, de posibilidades no resulta explicativa, ya que la noción de posibilidad requiere tanto ser elucidada como el propio concepto de propensión.

\footnotetext{
${ }^{7}$ Roberto Torretti elabora esta idea en 1990.
} 
Coincido con los propensistas, y otros autores, en que el concepto de posibilidad es básico; ${ }^{8}$ creo, sin embargo, que podemos caracterizarlo, saliendo de ese grupo de conceptos de azar, si atendemos a la estructura probabilista de las teorías de la física.

La idea germen de la que parto es la idea general de Leibniz de que la probabilidad es posibilidad con grados. Específicamente, interpreto los enunciados probabilistas en referencia a las posibilidades de los diferentes estados alternativos que un sistema físico puede adoptar, dadas ciertas condiciones iniciales, relativamente a una teoría física que describe el sistema.

El concepto cualitativo de posibilidad, sostenemos, está relacionado con el concepto cuantitativo de probabilidad - el cual está caracterizado formalmente por la teoría matemática de la probabilidad - de tal manera que, al menos en algunos casos, podemos representar al primero en referencia al segundo, para justificar la aseveración de que una expresión como "la probabilidad del suceso singular $e$ " significa "el grado de posibilidad del suceso singular $e^{\text {", }}$ en el contexto de una teoría física con estructura probabilista. En particular, mantengo que las probabilidades cuánticas pueden concebirse como las medidas de las posibilidades físicas de sucesos singulares en sistemas cuánticos, relativamente a la propia teoría cuántica.

Similarmente a Suppes, quien sostiene que la definición de causa genuina debe relativizarse a un marco conceptual ya que "No hay ni metafísica ni científicamente un último concepto de causa genuina" (1982, p. 206), mantengo que la noción de posibilidad física tiene que ser relativizada a un marco teórico, descartando cualquier concepto absoluto de posibilidad real como el popperiano.

Antes de exponer el concepto de posibilidad física propuesto, consideraré la "ontología" adecuada para poder predicar posibilidades físicas de sucesos singulares. Para ello parto de la siguiente noción de dispositivo o sistema azaroso debida a Ian Hacking: "un dispositivo azaroso [chance set-up] es un aparato

${ }^{8}$ También en otras interpretaciones de la probabilidad, como la frecuencial y la clásica-laplaceana, se recurre a alguna noción de posibilidad en tanto que suponen un conjunto de sucesos posibles, el llamado "espacio de sucesos". 
o parte del mundo en el cual se podrían realizar uno o varios ensayos, experimentos u observaciones; cada ensayo debe tener un único resultado, el cual es un miembro de la clase de resultados posibles" (1965, p. 13).

Ahora bien, como un dispositivo o sistema se conceptualiza como azaroso desde un marco teórico, añado que tanto los estados de los sistemas como los ensayos realizados en ellos sean descriptibles por una teoría física $\mathbf{T}$, y que la clase de los resultados posibles sea especificable por la propia teoría T. Al resultado que arroje un ensayo particular, efectuado en un dispositivo azaroso, lo llamaré un suceso singular, usando el término "ensayo" en un sentido amplio que incluye mediciones, experimentos y observaciones.

De esta manera, la noción de suceso singular es relativa a una teoría en cuyo lenguaje se describen los ensayos efectuados en los sistemas físicos, así como sus estados y evoluciones.

Una noción probabilista de la descripción de un estado físico que nos sirva de guía para caracterizar la noción de "suceso físicamente posible relativamente a $\mathbf{T}$ " es enunciada por Beltrametti y Cassinelli como sigue:

Por una descripción estática de un sistema físico queremos decir las reglas que asignan objetos matemáticos específicos a los estados y a las cantidades físicas del sistema, y las prescripciones para calcular la distribución de probabilidad de los valores posibles de toda cantidad física cuando el estado del sistema está dado. (1981, p. 3)

Podemos caracterizar ahora el concepto de posibilidad física, relativo a una teoría física $\mathbf{T}$, enunciando dos condiciones. La primera establece que el estado inicial de un dispositivo o sistema físico azaroso sea descriptible por la teoría T; es decir, que los valores que pueden asignarse a las cantidades físicas del sistema puedan ser especificados por T. Con ello se descartan como físicamente posibles las descripciones de estado de los sistemas que asignan valores que no están dentro del rango de definición de las cantidades físicas involucradas (por ejemplo, en mecánica clásica, descripciones que asignen a un cuerpo una 
masa no positiva o, en teoría de la relatividad, descripciones que asignen a una partícula elemental una velocidad mayor a la velocidad de la luz). La segunda condición establece que las descripciones de los sucesos físicos singulares, como resultados finales de la evolución del sistema, respondan a las prescripciones de la teoría $\mathbf{T}$.

De esta manera, diremos que un suceso singular $e$ en un sistema $\boldsymbol{S}$ es fisicamente posible respecto de una teoría $\mathbf{T}$ si

(1) el estado inicial del sistema $\boldsymbol{S}$ es descriptible por $\mathbf{T}$; y

(2) la descripción del suceso $e$, como resultado de un ensayo realizado en $\boldsymbol{S}$ (o de una evolución de $\boldsymbol{S}$ ), es prescriptible por las leyes de $\mathbf{T}$.

La condición (1) delimita los estados iniciales que un dispositivo o sistema físico puede asumir mientras que la condición (2) especifica, una vez establecido un estado inicial, los resultados posibles que el sistema físico puede adoptar en un ensayo efectuado en él; todo ello relativamente a una teoría $\mathbf{T}$ de la física.

Desde un punto de vista formal, este concepto de posibilidad física es más fuerte que el que asumen implícitamente algunos propensistas, como Giere. El de ellos supone compatibilidad con las leyes científicas, en el sentido lógico de que las afirmaciones de que algunos sucesos físicos singulares son posibles no resulten contradictorias con esas leyes. El concepto aquí elaborado requiere, por supuesto, la compatibilidad lógica con las leyes de la física, pero también precisa que las aseveraciones de la posibilidad física de sucesos singulares sean predicciones de las teorías físicas, en un sentido menos estrecho que el de la implicación lógica. Nuestra caracterización de la posibilidad física involucra que un suceso singular $s$ es físicamente posible relativamente a $\mathbf{T}$ si la descripción de $s$ es derivable de las leyes de $\mathbf{T}$, en un sentido amplio de "derivable" que abarca inferencias deductivas, derivaciones matemáticas (análisis matemático) y, en particular, cálculos probabilistas; esto depende del tipo de estructura de la teoría $\mathbf{T}$ en consideración. 
Podemos ilustrar nuestro concepto con un tipo de sistema de la mecánica cuántica. En esa teoría, el espín, o impulso angular intrínseco, se considera una propiedad "interna" de ciertas partículas, una propiedad como la masa o la carga eléctrica, que es medible. Su medición se representa por un vector con valores para las tres coordenadas. Un sistema físico, con una partícula, se define por los valores que corresponden a la proyección del espín de la partícula en las tres componentes: $S x, S y$ y $S z$. Ahora, según esa teoría, en un sistema compuesto por un electrón, esa magnitud sólo puede adoptar dos estados iniciales, con valores propios $1 / 2 \mathrm{y}-1 / 2$. Y dado uno de esos dos estados posibles, con el espín del electrón orientado en cualquier dirección, la mecánica cuántica prescribe qué resultados son físicamente posibles en un proceso de medición, es decir, qué valores puede adoptar el espín si se somete a una medición.

Por ejemplo, si el aparato de medición detecta la proyección del espín en dirección vertical y el valor inicial del espín en dirección horizontal es $1 / 2$, la mecánica cuántica prescribe que sólo son posibles dos resultados, con valores $1 / 2$ y $-1 / 2$ con probabilidades iguales. Si el espín es orientado, en las mismas condiciones, a 45 grados, esa teoría prescribe igualmente que esos dos valores son posibles, permitiendo calcular sus probabilidades, a saber: 0.85 para el valor $1 / 2$ y 0.15 para el valor $-1 / 2$. Así, ese tipo de sistema cuántico ejemplifica nuestro concepto de posibilidad física.

Este concepto de "suceso físicamente posible respecto de T" es un concepto general que da cabida a graduaciones de las posibilidades físicas, que dependen del tipo de teoría física — determinista o probabilista - que esté en el lugar de la variable $\mathbf{T}$.

Las prescripciones de las leyes de teorías deterministas, como la mecánica newtoniana, no gradúan la posibilidad, ya que predicen que, dadas ciertas condiciones iniciales de un sistema, un único suceso puede acontecer. Por ello, la posibilidad resulta ser un concepto de clase: dado un estado inicial de un sistema de la mecánica clásica, la ocurrencia de un suceso específico está determinada; el único suceso físicamente posible es ése (con 
probabilidad igual a la unidad), cualquier otro suceso distinto es físicamente imposible (con probabilidad nula).

Cuando en nuestra caracterización de la noción de posibilidad física relativa a teorías, la teoría $\mathbf{T}$ es la mecánica newtoniana, la consecuencia que se obtiene es, pues, que el de posibilidad es un concepto de todo o nada, sin grados. Esto se debe a que bajo esa concepción determinista del mundo físico, no hay lugar para sucesos contingentes, sucesos que pueden ocurrir o dejar de ocurrir, dadas circunstancias específicas.

En cambio, en teorías físicas con estructura probabilista - como la mecánica estadística y la mecánica cuántica-, las prescripciones ponderan la posibilidad de ocurrencia de los distintos sucesos contingentes que se asocian, como resultados experimentales de un sistema azaroso, a un estado inicial del mismo. En otras palabras, en ese tipo de teorías, el azar físico - sea epistémico u objetivo - como manifestación de las posibilidades físicas de los estados finales alternativos de los sistemas que estudian, es expresado por prescripciones probabilistas, las cuales atribuyen un grado a esas posibilidades.

En el caso de la mecánica clásica estadística, el azar involucrado podría ser epistémico —un reflejo de nuestro desconocimiento parcial de condiciones objetivas dadas-, pero en la mecánica cuántica, el carácter objetivo del azar físico encuentra soporte en que las descripciones probabilistas son irreductibles a descripciones clásicas con variables ocultas locales, de acuerdo con los resultados obtenidos por Alain Aspect y sus colegas ${ }^{9}$ en experimentos tipo Einstein, Podolsky y Rosen, en referencia al teorema de Bell.

Si en nuestra noción de posibilidad física, la teoría $\mathbf{T}$ en cuestión es la mecánica cuántica, obtenemos que las prescripciones emitidas sí atribuyen grados objetivos a los varios sucesos físicamente posibles.

Veamos esto en nuestro anterior ejemplo cuántico. En él podemos ver claramente que bajo ciertas condiciones específicas, cuando el electrón está orientado a 90 grados en relación con

${ }^{9}$ Aspect, Dalibard y Roger 1982. 
el detector del espín y el valor inicial de éste es $1 / 2$, la teoría cuántica asigna la probabilidad 0.5 a cada uno de sus dos valores propios mientras que si, en las mismas condiciones, la orientación del electrón es de 45 grados, las probabilidades que asigna a los valores propios del espín, son desiguales, a saber 0.85 para el valor $1 / 2$ y 0.15 para el valor - 1/2. De esta manera, esa teoría expresa los grados de posibilidad de los dos resultados alternativos de forma cuantitativa con enunciados probabilistas.

La situación anterior muestra que la cuestión de que si la noción de posibilidad física admite grados o no depende de la teoría T que estemos considerando. Esta consecuencia de la noción de posibilidad física aquí propuesta es aceptable, porque solamente desde una teoría física podemos decir si ciertos sucesos físicos son posibles o no y, en caso de que lo sean, en qué grado lo son. No podemos atribuir a un conjunto de sucesos físicos alternativos grados de posibilidad — sean subjetivos, epistémicos u objetivos - de manera independiente de cualquier teoría de la física y, por ello, son las mismas teorías las que deciden sobre la cuestión de la graduación de la posibilidad.

Si, además, reconocemos el carácter irreductible de las probabilidades cuánticas, tenemos que los grados de las posibilidades físicas de los sucesos cuánticos no son epistémicos sino objetivos; y están definidos por los estados de los sistemas cuánticos. Hay una noción de azar físico que nos permite capturar conceptualmente el tipo de azar que está involucrado por el carácter irreductible de los enunciados probabilistas cuánticos, y que Sergio Martínez ha caracterizado en estos términos:

Un azar objetivo no epistémico tiene que expresarse como la descripción de un aspecto de la estructura física del mundo, incorporado en la descripción de estado, según una teoría fundamental de la física (independientemente de las limitaciones de nuestra manera de observarlo o conocerlo que puedan existir). A este tipo de azar objetivo le llamamos azar sistémico. Es un tipo de azar objetivo que es intrínseco a la descripción de estado que la teoría utiliza. (1991, pp. 139-140) 
La teoría cuántica es un caso especial de teoría fundamental (o completa) con estructura probabilista. Hughes la considera inherentemente probabilista porque "cada uno de sus principios [de superposición e incertidumbre] implica que hay estados puros que asignan a los resultados de ciertos experimentos, probabilidades distintas a uno y a cero" (1989, p. 108). Más bien podemos considerarla irreductiblemente probabilista puesto que las probabilidades con valores no extremos implicadas por esos principios cuánticos no son, como ya hemos mencionado, reductibles a descripciones deterministas, clásicas.

La interpretación objetiva de la probabilidad aquí propuesta descansa sobre dos tesis. La primera, de índole ontológica, asevera que el mundo físico es tal que dadas ciertas circunstancias específicas, pueden acontecer varios sucesos alternativos. La otra, de naturaleza epistemológica, afirma que algunas teorías de la física nos proporcionan medios para graduar objetivamente la posibilidad de que acontezca cada uno de esos sucesos contingentes. La plausibilidad de estas dos aseveraciones proviene de que nos permiten capturar conceptualmente el comportamiento azaroso de cierto tipo de sistemas físicos como los cuánticos.

\section{Representación de las probabilidades cuánticas}

La clave para concebir las probabilidades como posibilidades con grados se encuentra en la clase de los resultados posibles de los ensayos en un sistema azaroso y en la asignación de valores de probabilidad a esos resultados, por parte de una teoría física. Esto nos permite interpretar los enunciados probabilistas como expresiones del grado de posibilidad física de dichos resultados, relativamente a la propia teoría.

Para justificar la aseveración anterior, mostraré que hay una representación implícita de la probabilidad en la teoría cuántica, en términos de una noción cualitativa de posibilidad que se ajusta al concepto de posibilidad física propuesto. La representación no es general; su alcance son magnitudes con espectro discreto de valores propios, como el espín de los electrones o la polarización de los fotones, mas no aquellas con espectro continuo, como la posición. Pero esta representación es signifi- 
cativa porque son precisamente ese tipo de magnitudes las que exhiben patentemente la irreductibilidad de las probabilidades cuánticas.

Para aplicar el concepto de probabilidad a esa teoría se precisa, como se sabe, modificar el álgebra de Boole subyacente a la formulación estándar o de Kolmogorov de los espacios de probabilidad, ya que la estructura algebraica de un conjunto de sucesos cuánticos no es booleana, por no ser distributiva. Entre las varias opciones de estructuras algebraicas candidatas, como la de retículo ortomodular (no distribuitivo), he elegido la de ortoálgebra por ser, tal vez, la más amplia. Presento su definición enseguida.

Definición A. Una estructura $<\mathbf{C}, \perp, \oplus, \perp, \mathbf{0}, \mathbf{1}>$ es una ortoálgebra si $\mathbf{C}$ es un conjunto con elementos destacados $\mathbf{0}$ y $\mathbf{1}, \perp$ es una relación binaria en $\mathbf{C}, \oplus$ es una operación binaria parcial en $\mathbf{C}$ tal que $a \oplus b$ existe si y sólo si $a \perp b,{ }^{\perp}$ es una relación unaria en $\mathbf{C}$, y para todo $a$ y $b$ en $\mathbf{C}$ :

(1) si $a \perp b$ entonces $b \perp a$ y $a \oplus b=b \oplus a$;

(2) $a \perp \mathbf{0}$ y $a \oplus \mathbf{0}=a$;

(3) $a \perp a^{\perp}$ y $a \oplus a^{\perp}=\mathbf{1}$;

(4) $a \perp a^{\perp} \oplus b$, sólo si $b=\mathbf{0}$;

(5) $a \perp a \oplus b$, sólo si $a=\mathbf{0}$;

(6) si $a \perp b$ entonces $a \perp(a \oplus b)^{\perp}$ y $b^{\perp} \perp a \oplus(a \oplus b)^{\perp}$. $^{10}$

$\perp$ es la relación simétrica de ortogonalidad, $\oplus$ representa la suma ortogonal, $\mathrm{y}^{\perp}$ denota la operación de complementación.

Ahora podemos definir una noción adecuada de espacio de probabilidad como sigue.

Definición B. Una estructura $\langle\mathrm{X}, \mathrm{S}, p\rangle$ es un espacio (finito) de probabilidad generalizada si y sólo si $\mathrm{X}$ es un conjunto finito de sucesos, $\mathrm{S}$ forma una ortoálgebra con conjunto base $\mathrm{X}$ y $p$ es una función de $\mathrm{S}$ a $[0,1]$ tal que

${ }^{10}$ Estos axiomas se deben a Hardegree y Frazer, cfr. Hughes 1989, p. 220. 
(1) $p(\mathbf{0})=0 ; p(\mathbf{1})=1 \mathrm{y}$

(2) para todo $x$ e $y$ en $\mathrm{S}$, si $x \perp y$ entonces $p(x \oplus y)=$ $p(x)+p(y)$.

Ahora bien, en las formulaciones estándar de la mecánica cuántica se usan espacios de Hilbert para describir los sistemas físicos; se asocia a estos un espacio (de dimensión apropiada) sobre el campo de los números complejos. Los estados puros de los sistemas cuánticos se representan entonces por vectores $\mathbf{v}$ y las cantidades observables $A$ por determinado tipo de operadores $\mathbf{A}$ en el espacio. Asimismo, a estos operadores se les asocia un subconjunto $\Delta$ de la línea real $\Re$, llamado el espectro de $A$, el cual contiene los valores posibles del observable $A$ (propiamente, $\Delta$ es un elemento de un conjunto de Borel en $\Re$ ).

La teoría cuántica prescribe la probabilidad de que el resultado de una medición del observable $A$ esté en el intervalo $\Delta$, estando el sistema en un estado (preparación o situación experimental) v. Podemos considerar el hecho de que el resultado de la medición de un observable $A$, dado un estado $\mathbf{v}_{i}$ del sistema, sea un $a_{i}$ determinado en $\Delta$ como un suceso singular cuántico, expresado por $\left(A \mid \mathbf{v}_{i}\right)=a_{i}$. En este sentido decimos que la mecánica cuántica prescribe la probabilidad de tales sucesos singulares, condicionales a un estado inicial, método de preparación o experimento. Es decir, establece los valores de la probabilidad de $\left(A \mid \mathbf{v}_{i}\right)=a_{i}$ o, como es usual escribirlo: $p_{\mathbf{v} i}(A$, $a_{i}$ ). Esto encuentra una buena ilustración en el caso de observables con vectores propios [eigen] y valores propios [eigen], como el espín $E$, donde tenemos, por ejemplo, que: $p_{y+}\left(E_{y}, 1 / 2\right)$ $=1, p_{y-}\left(E_{y}, 1 / 2\right)=0$ y $p_{x+}\left(E_{y}, 1 / 2\right)=0.5$. Vemos aquí que se asignan probabilidades a los sucesos singulares $\left(E_{y} \mid \mathrm{y}+\right)=1 / 2$, $\left(E_{y} \mid \mathrm{y}-\right)=1 / 2$ y $\left(E_{y} \mid x+\right)=1 / 2$.

Adopto aquí esta formulación usual (refiriéndome sólo a espacios de dimensión finita, bidimensional) a fin de demostrar un teorema de representación para cierto tipo de enunciados probabilistas cuánticos, satisfaciendo la demanda de Suppes.

La base de esa representación es el conjunto $\mathrm{X}$ de resultados posibles, sucesos singulares de la forma $\left(A \mid \mathbf{v}_{i}\right)=a_{i}$, asociado 
a un tipo particular de medición de $A$, y una función $\mu$ que pondera la posibilidad de esos resultados.

A cada resultado singular atómico ${ }^{11}\left(A \mid \mathbf{v}_{i}\right)=a_{i}$ asociamos el subespacio unidimensional o "rayo" del espacio complejo $C^{2}$, denotado por $\mathbf{L}_{i}^{A}$ que contiene a $\mathbf{v}_{i}$. Suponemos que los subespacios unidimensionales asociados a los resultados singulares del conjunto $\mathrm{X}$ son mutuamente ortogonales y que el espacio entero es igual a la expansión [span] del conjunto de esos subespacios. Por lo primero tenemos que los resultados singulares del conjunto $\mathrm{X}$ son mutuamente excluyentes, mientras que por lo segundo que son conjuntamente exhaustivos.

El conjunto base $\mathrm{X}$ puede ser ampliado a un conjunto $\mathrm{S}$, con $\mathrm{S} \subseteq \operatorname{Pot}(\mathrm{X})$, por las operaciones usuales de unión, intersección y complementación (al suceso atómico $\mathbf{L}_{i}^{A}$ en $\mathrm{X}$ le corresponde el conjunto singular $\left.\left\{\mathbf{L}_{i}^{A}\right\}\right)$.

Para nuestros propósitos, $\mathrm{S}$ puede ser dotado de la estructura correspondiente a una ortoálgebra de la siguiente manera. Dos sucesos $\mathbf{L}_{i}^{A}$ y $\mathbf{L}_{j}^{A}$ son ortogonales, $\mathbf{L}_{i}^{A} \perp \mathbf{L}_{j}^{A}$, si su intersección es el conjunto vacío. Este conjunto representa el suceso nulo, identificado con el componente $\mathbf{0}$ de la ortoálgebra. La suma ortogonal de un par de sucesos $\mathbf{L}_{i}^{A}$ y $\mathbf{L}_{j}^{A}, \mathbf{L}_{i}^{A} \oplus \mathbf{L}_{j}^{A}$, se equipara con su unión conjuntista. Como los sucesos en $\mathrm{S}$ están asociados a un experimento en particular, la operación $\oplus$ no está definida para sucesos asociados a experimentos diferentes y, así, es una operación parcial referida a un $\mathrm{S}$. El suceso "cierto" X es igual a $\cup_{i}\left\{\mathbf{L}_{i}^{A}\right\}$ (relativamente a un experimento o medición) y se identifica con el componente $\mathbf{I}$ de la ortoálgebra. El complemento $\mathbf{L}_{i}^{A^{\perp}}$ de $\mathbf{L}_{i}^{A}$ es el complemento conjuntista relativo a X.

Los conjuntos recién especificados tienen correlatos en los espacios de Hilbert. De nuevo, los resultados experimentales se representan por los subespacios $\mathbf{L}_{i}^{A}$, los cuales corresponden a los sucesos singulares $\left\{\mathbf{L}_{i}^{A}\right\}$. Además, al conjunto $\mathrm{X}$, que representa el suceso "cierto", le corresponde el espacio $V$ y al suceso nulo el subespacio cero $\mathbf{L}_{0}$.

${ }^{11}$ Es decir, que no puede dividirse en un par de resultados alternativos. 
De esta manera, del conjunto $\mathrm{X}$ de los resultados posibles de un experimento, medición o método de preparación, se genera un conjunto $\mathrm{S}$ para formar una ortoálgebra. Es decir, la estructura $<\mathrm{S}, \perp, \oplus, \stackrel{\perp}{ }, \mathbf{0}, \mathbf{1}>$ es una ortoálgebra en la que, en particular, $\oplus$ es una operación parcial en $\mathbf{S}$ tal que $\mathbf{L}_{i}^{A} \oplus \mathbf{L}_{j}^{A}$ existe si y sólo si $\mathbf{L}_{i}^{A} \perp \mathbf{L}_{j}^{A}$.

La representación que se va a demostrar puede formularse por medio del siguiente teorema.

Teorema de representación. Sea X un conjunto (no vacío) finito de sucesos atómicos y que $\mathrm{S}$ forma una ortoálgebra con conjunto base $\mathrm{X}$. Sea $\langle\mathrm{X}, \mathrm{S}, p\rangle$ un espacio de probabilidad generalizada. Entonces existe una función $\mu$, con dominio en $\mathrm{X}$, tal que

$$
\mu_{\mathbf{v}}\left(\mathbf{L}_{i}^{A}\right) \text { si y sólo si } p_{\mathbf{v}}\left(A, a_{i}\right)^{12}
$$

que satisface los axiomas de la definición de espacio de probabilidad generalizada (Definición B).

Prueba. ${ }^{13}$ Definimos una función $\mu$, de ponderación de la posibilidad de los sucesos, con dominio en X, así:

$$
\mu_{\mathbf{v}}\left(\mathbf{L}_{i}\right)=\left|\mathbf{P}_{i}\right|^{2},
$$

donde $\mathbf{P}_{i}$ es un operador de proyección del vector $\mathbf{v}$ en un espacio de Hilbert.

Suponemos que (i) los vectores $\mathbf{v}_{i}$ están normalizados, como es el caso del vector-espín; (ii) que esos vectores $\mathbf{v}_{i}$ son vectores propios con un conjunto finito $\Delta$ asociado de valores propios $a_{i}$, y (iii) que los operadores $\mathbf{P}_{i}$ son proyectores, por ello, hermitianos e idempotentes.

Antes que nada, nótese que como los vectores $\mathbf{v}$ son normalizados tenemos que

${ }^{12}$ Nótese que esta última fórmula es equivalente a $p(A, \mathbf{v})=a_{i}$, la cual se refiere a la probabilidad de un suceso singular, el suceso de que la medición de la magnitud $A$, representada por el operador $\mathbf{A}$, estando el sistema en el estado (representado por) $\mathbf{v}$, arroje el resultado $a_{i}$, donde, por supuesto, $a_{i}$ está en el conjunto discreto $\Delta$ de resultados posibles.

${ }^{13}$ La demostración que sigue la he construido a partir de algunos capítulos del libro de Hughes 1989. 


$$
0 \leq \mu_{\mathbf{v}}\left(\mathbf{L}_{i}\right) \leq 1
$$

esto es, que el rango de la función $\mu$ es $[0,1]$ como se requiere.

Usando la ecuación cuántica $p\left(A, a_{i}\right)=<\mathbf{v} \mid \mathbf{P}_{i}^{A} \mathbf{v}>$ (la cual es la versión del algoritmo fundamental para espectros discretos de valores propios), probamos primero que $\mu_{\mathbf{v}}\left(\mathbf{L}_{i}^{A}\right)$ implica $p_{\mathbf{v}}\left(A, a_{i}\right)$ de la siguiente manera:

$$
\begin{aligned}
\mu_{\mathbf{v}}\left(\mathbf{L}_{i}^{A}\right) & =\left|\mathbf{P}_{i}^{A} \mathbf{v}\right|^{2} & & \\
& =<\mathbf{P}_{i}^{A} \mathbf{v} \mid \mathbf{P}_{i}^{A} \mathbf{v}> & & \text { (por definición de longitud) } \\
& =<\mathbf{v} \mid \mathbf{P}\left(\mathbf{P}_{i}^{A} \mathbf{v}\right)> & & \text { (hermeticidad) } \\
& =<\mathbf{v} \mid \mathbf{P}_{i}^{A} \mathbf{v}> & & \text { (idempotencia) } \\
& =p_{\mathbf{v}}\left(A, a_{i}\right) & & \text { (ecuación cuántica) } .
\end{aligned}
$$

La implicación inversa se demuestra análogamente.

Veamos ahora cómo se satisfacen los axiomas. La forma que adoptan esos axiomas, en el marco semántico aquí presentado, es la siguiente (dando por supuesto que se cumplen las condiciones para $\mathrm{X}$ y para $\mathrm{S})$ :

$\left(1^{\prime}\right) \mu_{\mathbf{v}}(\mathbf{0})=0 ; \mu_{\mathbf{v}}(\mathbf{1})=1 \mathrm{y}$

$\left(2^{\prime}\right)$ para cualquier par de subespacios unidimensionales $\mathbf{L}_{i}$ y $\mathbf{L}_{j}$ en $\mathrm{S}$, si $\mathbf{L}_{i} \perp \mathbf{L}_{j}$ entonces $\mu_{\mathbf{v}}\left(\mathbf{L}_{i} \oplus \mathbf{L}_{j}\right)=\mu_{\mathbf{v}}\left(\mathbf{L}_{i}\right)+$ $\mu_{\mathbf{v}}\left(\mathbf{L}_{j}\right)$.

Nótese que los rayos $\mathbf{L}_{i}$ y los operadores de proyección $\mathbf{P}_{i}$ están en una correspondencia uno a uno; en particular, al subespacio cero $\mathbf{L}_{0}$ le corresponde el proyector cero $\mathbf{P}_{0}$ y al espacio $V(=\mathrm{X})$ el proyector identidad $\mathbf{I}$.

Respecto de $\left(1^{\prime}\right)$ por la definición de $\mu$, primero, tenemos que $\mu_{\mathbf{v}}(\mathbf{0})=\mu_{\mathbf{v}}\left(\mathbf{L}_{0}\right)=\left|\mathbf{P}_{0} \mathbf{v}\right|^{2}=0$. Y, segundo, tenemos que $\mu_{v}$ (1) $=\mu_{\mathbf{v}}(V)=|\mathbf{I v}|^{2}=|\mathbf{v}|^{2}=1$.

En relación con $\left(2^{\prime}\right)$ tenemos que si $\mathbf{P}_{i}$ y $\mathbf{P}_{j}$ proyectan subespacios ortogonales $\mathbf{L}_{i}$ y $\mathbf{L}_{j}$, entonces ellos son ortogonales entre sí. Denotando $\mathbf{L}_{i} \oplus \mathbf{L}_{j}$ por $\mathbf{L}_{k}$, por el teorema de Pitágoras obtenemos 


$$
\left|\mathbf{P}_{i} \mathbf{v}\right|^{2}+\left|\mathbf{P}_{j} \mathbf{v}\right|^{2}=\left|\mathbf{P}_{k} \mathbf{v}\right|^{2},
$$

que concuerda con $\left(2^{\prime}\right)$ haciendo las substituciones correspondientes.

La existencia de la representación anterior tal vez contesta el comentario de Hughes - en referencia al problema de la interpretación de la probabilidad - de que las probabilidades de la mecánica cuántica, al menos las que se refieren a estados puros, son probabilidades objetivas. Hughes, quien rechaza las propensiones calificándolas de entidades misteriosas, no propone una solución a ese problema, sólo sostiene que esas probabilidades "son inherentes en el mundo y no simplemente reflejan los grados de creencia de un observador", por eso las considera objetivas (1989, p. 218). Nuestra interpretación, quizá, pueda subsanar ese tipo de carencia, respondiendo a intuiciones filosóficas como la de Hughes de que las probabilidades son inherentes en el mundo.

\section{Conclusiones}

El carácter objetivo, no epistémico, de la presente interpretación de la probabilidad depende de que la teoría física en cuestión, con estructura probabilista, sea completa en un sentido pertinente, esto es, que no existan magnitudes "ocultas" cuya incorporación a la teoría convierta a sus descripciones y predicciones probabilistas en enunciados clásicos, deterministas.

En el caso de la teoría cuántica, contamos con los resultados de los experimentos efectuados por Aspect, que constatan la irreductibilidad de las probabilidades cuánticas a enunciados no probabilistas que incorporen variables ocultas locales. Podría suceder que tal irreductibilidad de las probabilidades cuánticas no fuera un resultado definitivo, puesto que la posibilidad de la existencia de magnitudes físicas, hasta ahora desconocidas, que tuvieran efectos no locales - efectos que se propagaran con una velocidad mayor que la de la luz - no está descartada por esos experimentos, ya que en éstos se suponen sólo efectos locales. Así, la existencia de variables ocultas no locales, podría significar un reto al carácter no epistémico de los enunciados probabilistas de la teoría cuántica. 
El sentido objetivo que podamos atribuir a la interpretación de las probabilidades como grados de posibilidades físicas relativas a una teoría depende de que podamos adjudicar, a los enunciados probabilistas de la teoría en cuestión, un carácter irreducible. Esto último da la razón por la que no podemos establecer de antemano si cabe interpretar los enunciados probabilistas de una teoría física dada de una manera objetiva, no epistémica, en términos de posibilidades físicas.

La propuesta básica y general ofrecida aquí consiste en un concepto de posibilidad física relativo a una teoría, en cuyo contexto se pueden interpretar las probabilidades; mas la cuestión de si podemos o no atribuir a los enunciados probabilistas de una teoría particular un sentido objetivo — que no dependa de nuestras limitaciones, objetivas o subjetivas, de conocerestá fuera del alcance de esta propuesta y es una cuestión que se debe examinar en cada caso particular de teoría.

\section{BIBLIOGRAFÍA}

Aspect, Alain, J. Dalibard y G. Roger, 1982, "Experimental Test of Bell's Inequalities Using Time-Varing Analyzers", Physical Review Letters, 49, pp. 1804-1807.

Beltrametti, Enrico G. y G. Cassinelli, 1981, The Logic of Quantum Mechanics, Addison-Wesley, Reading, Mass.

Galavotti, Maria Carla, 1987, "Comments on Patrick Suppes 'Propensity interpretations of probability", Erkenntnis, 26, pp. 359-368.

Giere, Ronald, 1976, "A Laplacean Formal Semantics for Single-Case Propensities", Journal of Philosophical Logic, 5, pp. 321-353.

— 1973, "Objective Single-Case Probabilities and the Foundations of Statistics", Patrick Suppes et al. (comps.), Logic, Methodology and the Philosophy of Science, IV, North Holland, Elsevier, pp. 467-483.

Hacking, Ian, 1965, The Logic of Statistical Inference, Cambridge University Press, Cambridge.

Humphreys, Paul, 1985, "Why Propensities Cannot Be Probabilities", The Philosophical Review, vol. XCIV, no. 14, pp. 557-570.

Hughes, R.I.G., 1989, The Structure and Interpretation of Quantum Mechanics, Harvard University Press, Harvard, Mass. 
Martínez, Sergio, 1991, "El azar en la mecánica cuántica: de Bohr a Bell”, Crítica, vol. XXIII, no. 69, pp. 137-154.

Popper, Karl, 1990, Un mundo de propensiones, trad. José Miguel Esteban Cloquell, Tecnos, Madrid, 1992.

_- 1982a, Realismo y el objetivo de la ciencia, trad. Marta Sansigre Vidal, Tecnos, Madrid, 1984.

- 1982 b, El universo abierto, trad. Marta Sansigre Vidal, Tecnos, Madrid, 1984.

- - 1982c, Teoría cuántica y el cisma en física, trad. Marta Sansigre Vidal, Tecnos, Madrid, 1984.

— 1 1959, "The Propensity Interpretation of Probability", British Journal of the Philosophy of Science, 10, pp. 25-42.

, 1957, "The Propensity Interpretation of the Calculus of Probability and the Quantum theory", en S. Körner (comp.), Observation and Interpretation in the Philosophy of Physics", Butterworth, Londres, pp. 65-70. [Versión en castellano: "Propensiones, probabilidades y la teoría cuántica”, en D. Miller (comp.), Popper. Escritos selectos, trad. Sergio René Madero Báez, Fondo de Cultura Económica, México, 1995.]

Salmon, Wesley, 1979, "Propensities: A Discussion Review", Erkenntnis, 14, pp. 183-216.

Suppes, Patrick, 1987, "Propensity Representations of Probability", Erkenntnis, 26, pp. 335-358.

—. 1984, Probabilistic Metaphysics, Basil Blackwell, Nueva York/Oxford.

__ 1982, "Problemas del análisis causal en las ciencias sociales", en José Luis Rolleri (comp. y trad.), Estudios de filosofía y metodología de la ciencia, Alianza Universidad, Madrid, 1988, pp. 203-223.

Torretti, Roberto, 1990, Creative Understanding, University of Chicago Press, Chicago.

Van Fraassen, Bas, 1980, La imagen científica, trad. Sergio Martínez, Paidós/Instituto de Investigaciones Filosóficas-UNAM, México, 1996.

Recibido el 8 de enero de 2002; revisado el 20 de junio de 2002; aceptado el 31 de julio de 2002 